Methods Up to 2113 participants from the MRC National Survey of Health and Development, a British cohort study followed since birth in 1946, were included in analyses. Stressful life events, including divorce and death of a loved one, between ages 36 and 60-64 were counted (max score: 32). Mastery (Pearlin Mastery Scale), symptoms of anxiety and depression (General Health Questionnaire-28), wellbeing (Warwick-Edinburgh Mental Wellbeing Scale) and fear of falling (yes/no) were ascertained at age 68. Standing balance was assessed at age 69 using the one-legged stand test with eyes closed (max: 30 seconds). Linear regression models were used to examine associations between each psychological factor and log-transformed balance in sex and fully-adjusted models. Adjustments were made for height, body mass index, socioeconomic position, physical activity, smoking history, osteoarthritis, diabetes, cardiovascular events and presence of respiratory symptoms at age 68-69.

Results In sex-adjusted models, all five psychological factors were associated with balance performance; these associations were maintained after adjustment for covariates. In fullyadjusted models: for every additional stressful life event, individuals had a $2 \%(95 \%$ CI $0.3 \%$ to $3 \%)$ decrease in balance time; there was a $2 \%(1 \%-2 \%)$ increase in balance time for every 1 point increase in mastery; for every SD increase in depressive symptoms and wellbeing, there was a 5\% (2\%-8\%) decrease and $5 \%(2 \%-9 \%)$ increase in balance time respectively; those who reported fear of falling had 17\% (9\%-25\%) lower balance time when compared with those who had no fear.

Conclusion Experiencing a greater number of stressful life events, greater levels of anxiety and depression, lower wellbeing, lower mastery and fear of falling were all associated with poorer balance performance at age 69. These associations were robust to adjustment for a wide range of potential confounders. This suggests that psychological factors may be suitable targets for intervention aimed at reducing balance impairments in older adults. Future research should consider how these psychological factors interrelate and if they operate on similar pathways.

\section{OP28 COMMUNITY CULTURAL ENGAGEMENT AND INCIDENT DEPRESSION IN OLDER ADULTS}

'D Fancourt* ${ }^{2} \mathrm{U}$ Tymoszuk. 'Department of Behavioural Science and Health, University College London, London, UK; ${ }^{2}$ Department of Epidemiology and Public Health, University College London, London, UK

\subsection{6/jech-2018-SSMabstracts.28}

Background 1 in 4 older adults in the UK is affected by depression, which is associated with a range of physical health outcomes. So there is a recognised need to identify behavioural interventions that might protect against the development of depression. Over the past decade, research has demonstrated the effects of community cultural engagement (which combines a number of protective factors including social interaction, cognitive stimulation and gentle physical activity) on the treatment of depression. This has included studies on active cultural engagement (such as singing, dancing and crafts) and receptive cultural engagement (including visiting museums and galleries). But as yet there is little research on community cultural engagement and prevention of depression. Consequently, this study aimed to explore whether cultural engagement in older adults is associated with a reduced risk of developing depression over the following decade.

Methods This study used data from the English Longitudinal Study of Ageing: specifically data from Wave 2 (2004/2005) across every biennial wave through to Wave 7 (2014/2015); a total of 6 waves and a decade of data. We selected individuals who had no indication of depression at baseline using the Centre for Epidemiologic Studies Depression Scale (CES-D; score of $<3$ ) and used logistic regression models to explore the odds of developing depression at any wave over the following 10 years in relation to how often people engaged in community cultural activities (including visiting museums/galleries, attending the theatre/concerts/opera, and going to the cinema). For all analyses, we controlled for all identified socio-demographic confounders, health confounders (including sensory impairment, chronic conditions and pain) and social confounders (including other forms of social/civic engagement).

Results There was a dose-response relationship between frequency of cultural engagement and the risk of developing depression independent of socio-demographic, health-related and social confounders. This equated to a $34 \%$ lower risk of developing depression for people who attended every few months $(\mathrm{OR}=0.66,95 \% \mathrm{CI} 0.46$ to $0.96, \mathrm{p}=0.03)$ and a $50 \%$ lower risk for people who attended once a month or more $(\mathrm{OR}=0.5,95 \% \mathrm{CI} 0.33$ to $0.76, \mathrm{p}=0.001)$. Results were robust for sensitivity analyses exploring reverse causality and alternative CES-D thresholds.

Conclusion The results presented here suggest that cultural engagement is an independent risk-reducing factor for the development of depression in older age. In light of these data, it is suggested that behavioural intervention studies are designed to test further whether community cultural engagement can causally decrease the risk of developing depression.

\section{OP29 A MEASURE TO IMPROVE ASSESSMENT AND MANAGEMENT OF SYMPTOMS AND CONCERNS OF PEOPLE WITH DEMENTIA IN CARE HOMES: DEVELOPMENT AND MIXED METHODS EVALUATION}

${ }^{1} \mathrm{C}$ Ellis-Smith*, 'IJ Higginson, ${ }^{1,2} \mathrm{CJ}$ Evans. ${ }^{1}$ Cicely Saunders Institute of Palliative Care, Policy and Rehabilitation, King's College London, London, UK; ${ }^{2}$ Adult Nursing, Sussex Community NHS Foundation Trust, Brighton, UK

\subsection{6/jech-2018-SSMabstracts.29}

Background Detection and management of physical symptoms and psychosocial concerns for people with dementia is essential to reduce suffering. Measures used in routine care can support assessment and change care processes to improve outcomes. We aimed to develop and conduct a feasibility and process evaluation of the mechanisms of action (MOA), acceptability and implementation requirements of the Integrated Palliative Care Outcome Scale for Dementia (IPOSDem) to improve comprehensive assessment and management of symptoms and concerns of residents with dementia in care homes.

Methods A mixed methods interventional design informed by the MRC framework and underpinned by a theoretical model of expected MOA. Study conducted in three care homes involving residents with dementia, family, care staff and health professionals. IPOS-Dem V1 was developed from the validated IPOS through a scoping review of symptoms and concerns common in dementia. Two sequential phases followed. Phase 
one comprised focus groups/semi-structured interviews and cognitive interviews to evaluate content validity, comprehensibility and acceptability; and refinements to IPOS-Dem made. Phase two employed an embedded mixed methods design to evaluate MOA, feasibility and implementation requirements. Residents received IPOS-Dem as part of routine care for 12 weeks. Qualitative data included focus groups, semi-structured interviews and observations. Quantitative data comprised IPOS-Dem scores. Directed content analysis and descriptive statistics was used for qualitative and quantitative data analysis respectively, analysed separately and then integrated on key areas to inform a final theoretical model.

Results Phase one: 26 family, care staff and health professionals participated in focus groups/interviews, and 10 care staff in cognitive interviews. Five additional items were identified as important for content validity. Refinements to improve acceptability and comprehensibility included use of lay terms and item descriptors. Phase two: 32 residents received IPOSDem and 18 family, care staff and health professionals participated in focus groups, interviews and observations. Key MOA were improved collaborative assessment resulting in improved detection of symptoms and concerns; comprehensive 'picture of the person' which supported systematic record-keeping and monitoring, and facilitated communication between care staff, family, and health professionals. IPOS-Dem was perceived as easy to use and providing value to care, with the proportion of missing data decreasing from $2.1 \%$ at baseline to $1.1 \%$ at 12 weeks.

Conclusion IPOS-Dem is an acceptable and feasible measure to improve comprehensive assessment and management of symptoms and concerns in residents with dementia. A theoretical model of likely MOA and implementation requirements is presented. Further psychometric testing and effectiveness trial is required.

Funders Cicely Saunders International, Atlantic Philanthropies. This research was supported by the National Institute for Health Research (NIHR) Collaboration for Leadership in Applied Health Research and Care South London (NIHR CLAHRC South London) at King's College Hospital NHS Foundation Trust. The views expressed in this article are those of the author(s)and not necessarily those of the NHS, the NIHR, or the Department of Health and Social Care.

\section{Health services research}

\section{OP30 \#INTEGRATED CARE AT THE FRONTLINE: A PARTICIPATORY EVALUATION OF LOCALITY LEVEL MULTI-PROFESSIONAL TEAMS IN EAST LONDON}

M Lalani*, S Bussu, M Marshall. Primary Care and Population Health, University College London, London, UK

\subsection{6/jech-2018-SSMabstracts.30}

Background NHS England's new models of care (NCM) offer a platform for local partnerships between health and social care providers to reduce fragmentation of services and to support an integrated approach to care provision that is patientcentred and coordinated. The rationale behind these partnerships is to promote multi-professional working and integrated care, e.g. locality level multi-professional teams. These teams provide care coordination and case management for patients whose needs are most appropriately met by different health and social care professionals. The aim of the evaluation is to assess the enablers and barriers of implementing a multi-professional care model in primary care.

Methods The model of care presented here is being evaluated using a participatory approach to research: the Researcher in Residence (RiR). The researchers are embedded at the locality level and are using a formative, process-orientated approach employing primarily qualitative methods to gather data including participant observation, interviews, focus groups and documentary analysis. The RiR model places the researcher as a key member of the delivery team and enables co-creation of knowledge between researchers and practitioners, with the aim to increase opportunities for evidence to influence programme development. Furthermore, we have developed a maturity matrix tool which will enable the organisations participating in the evaluation to assess the extent of the development of the locality level teams over time.

Results Preliminary findings have revealed that a series of system enablers to promote integrated working have been introduced including efforts to co-locate health and social care staff. Whereas co-location might contribute to team building as evidenced by effective working between health professionals, it is not a panacea, and organisational development needs are evident in terms of organisational, cultural and professional issues, i.e. different management lines and organisational pressures, professional identity, trust, and accountability, compounded by rapid staff turnover and high numbers of locum staff.

Conclusion Frontline staff from both health and social care have demonstrated a desire to ensure delivery of joined up patient-centred care; interdisciplinary teams can potentially play a crucial role in driving greater care coordination. However, a plethora of policy initiatives resulting in continual reconfiguration of community health services while overlooking the same stumbling blocks that have continued to hamper previous efforts at strengthening integrated care may weaken outcomes once again. Better understanding of patterns of collaborations and integrated care pathways is crucial to identify frontline staff's organisational development needs and provide adequate support.

\section{OP31 TOWARDS UNDERSTANDING THE 'PARTNER' IN PARTNER NOTIFICATION FOR SEXUALLY TRANSMITTED INFECTION HEALTHCARE: MOVING BEYOND THE DICHOTOMY OF 'REGULAR' AND 'CASUAL' PARTNERS}

${ }^{1} \mathrm{M}$ Pothoulaki* ${ }^{*}{ }^{1} \mathrm{G}$ Vojt, ${ }^{2} \mathrm{~F}$ Mapp, ${ }^{2} \mathrm{CH}$ Mercer, ${ }^{1} \mathrm{CS}$ Estcourt, ${ }^{2} \mathrm{M}$ Woode-Owusu, ${ }^{3} \mathrm{~J}$ Cassell, ${ }^{2} \mathrm{~S}$ Wayal, ${ }^{4} \mathrm{M}$ Symonds, ${ }^{5} \mathrm{R}$ Nandwani, ${ }^{2} \mathrm{~J}$ Saunders, ${ }^{1} \mathrm{P}$ Flowers. ${ }^{1}$ School of Health and Life Sciences, Glasgow Caledonian University, Glasgow, UK; ${ }^{2}$ Institute of Global Health, University College London, London, UK; ${ }^{3}$ Department of Primary Care and Public Health, Brighton and Sussex Medical School, Brighton, UK; ${ }^{4}$ Barts Sexual Health Centre, Barts Health NHS Trust, London, UK; ${ }^{5}$ Sandyford Sexual Health Services, NHS Greater Glasgow and Clyde, Glasgow, UK

\subsection{6/jech-2018-SSMabstracts.31}

Background Partner notification (PN) is a key strategy for sexually transmitted infection (STI) management to reduce transmission and improve population health. It involves contacting sexual partners of people diagnosed with an STI and encouraging testing and treatment to prevent onward transmission, and re-infection. Current UK PN practice tends to conceptualise sexual partner types as 'regular' or 'casual'. However these terms do not sufficiently capture diverse sexual behavioural 